\title{
Aspirin Exacerbated Respiratory Disease and Nasal Polyps
}

MB Cupic ${ }^{1,2}$, S Savovic ${ }^{1,2}$, S Hromis ${ }^{1,3}$, SL Komazec ${ }^{1,2}$, M Savic ${ }^{2}$

\begin{abstract}
Objective: This study estimated the prevalence of patients with aspirin exacerbated respiratory disease (AERD) in the total group of patients with chronic rhinosinusitis with nasal polyps (CHRwNP), as well as a statistically significant difference between these two groups.

Material and method: By analyzing 150 patients with diagnoses of CHRwNP, a subgroup of patients with intolerance to aspirin and other NSAIDs with asthma was isolated.

Results: Out of 150 surveyed patients $57.3 \%$ had bronchial hyperreactivity, asthma was in $39.33 \%$ of patients and $25.3 \%$ had AERD. The average age of all examined patients was $52.83 \pm$ 12.28 years, while those with AERD were $47.24 \pm 13.35$. The ratio was 1: 1.7 in favor of women. At the initial endoscopic examination, $63.16 \%$ of patients were diagnosed with second degree and $36.84 \%$ with the third degree of polyps. After a year of therapy, we registered in $2.63 \%$ of patients first degree polyps in $60.53 \%$ second and in $36.84 \%$ third degree nasal polyps. During the year, with the first adequate therapy, $26.32 \%$ of patients required another round of a systemic corticosteroid therapy and $73.68 \%$ of the patients required three therapies.

Conclusion: CHRwNP in AERD, is the most severe form of nasal polyps, with statistically significant presence of difficult forms of polyps, as well as the need for larger number of repeated rounds of systemic corticosteroids during one year and the worst result of treatment with a large number of residual polyps, as well as a statistically large number of decision on further surgical treatment.
\end{abstract}

Keywords: Aspirin exacerbated respiratory disease (AERD), aspirin intolerance, chronicrhinosinusitis with polyps (CHRwNP), nasal polyps

From: ${ }^{1}$ Faculty of Medicine, University of Novi Sad, ${ }^{2}$ Clinical Center of Vojvodina, ENT Clinic, Novi Sad, and ${ }^{3}$ Institute for pulmonary diseases of Vojvodina, Sremska Kamenica, Serbia.

Correspondence: Dr M Čupić, Faculty of Medicine, University of Novi Sad, Clinical Centar of Vojvodina, ENT Clinic, HajdukVeljkova 1-9, Novi Sad, Serbia. E-mail: majabuljcik@gmail.com, MAJA.BULJCIK-CUPIC@mf.uns.ac.rs 


\section{INTRODUCTION}

Aspirin exacerbated respiratory disease (AERD) is a clinical syndrome of inflammation of the upper and lower respiratory tract that is difficult to control, characterized by eosinophilic chronic rhinosinusitis with polyps and bronchial asthma. Aspirin and other NSAIDs that inhibit cyclooxygenase-1 worsen this condition, causing severe nasal and asthmatic attacks. The characteristic of this syndrome is a typical sequence of symptoms the upper and lower respiratory tract. Rhinitis, as the first symptom of the disease, occurs after infection similar to flu, characterized by nasal dysfunction, followed by watery secretion of the nose, difficult breathing through the nose and sneezing. These symptoms become persistent, perennial and difficult to treat, with the later development of chronic rhinosinusitis with polyps. Asthma develops two to three years later. At the time of development of asthma, first and unexpected reactions to aspirin or other NSAIDsoccur, in patients who have previously tolerated it well $(1,2$, $3,4)$.

Chronic rhinosinusitis with polyps (CRSwNP) presents large heterogenic group diseases of the upper respiratory tract, with the different etiology and immunological pathway, but at the end all patients develop polyps in the nose (5). The main characteristic of nasal polyps in patients with AERD is a high degree of nasal polyps, which fully comply the nasal cavity, the bad and short-term response to medical and surgical therapy, a high recurrence rate and a very reduced quality of life $(6,7,8)$.

The AIM was to determine the prevalence of patients with AERD in the total group of patients with nasal polyps. To examine whether there is a statistically significant difference between patients with AERD and patients with CRSwNP without AERD regarding: the presence of allergies to inhaled allergens and findings of eosinophils in nasal secretions; the size of nasal 
polyps before and after the administration of corticosteroid therapy; the number of repetitions of required systemic corticosteroid therapy for one year, as well as decisions about patients' need for surgical treatment applied after corticosteroid therapy was applied for one year.

\section{MATERIALS AND METHODS}

This study was retrospective and prospective in character. Included one hundred and fifty patients with the diagnosis of CRSwNP, verified by the criteria of the European Rhinology Society and the American Academy of Otolaryngology and Head and Neck Surgery. Among them, a separate group of the patients with AERD was verified by a pulmonologist. The analysis was based on data obtained from the rhinological and allergy treatment protocols and therapeutic monitoring of the patient during one year.

The following data was analyzed: demographic data on patients with AERD, total number of patients suffering from sino-nasal polyposis, endonasal endoscopic findings, laboratory findings (eosinophils in the nasal discharge and nasal swab for bacteria and fungi), skin prick test with standard inhalatory allergens, history of the patient - whether he previously had nose surgery and paranasal sinus surgery. With endoscopic examination of the nose, the size of polyps was verified according to the consensus document adopted in Davos, classified into: 0 ${ }^{\circ}$ no evident presence of polyps in the nose, $1^{\circ}$ polyps confined to the middle nasal meatus, to the lower edge of the middle turbinate, $2{ }^{\circ}$ polyp out of the middle nasal meatus, limited to the lower edge of the inferior turbinate, that does not completely obstruct nasal cavity $3{ }^{\circ}$ polyp that completely obstructs nasal cavity. 
The number of repeated treatments with systemic corticosteroids and the final decision of the patient regarding the operation after one year of follow-up were analyzed from therapeutic protocols. The study was approved by the University Hospital of the Vojvodina/Faculty of Medical Sciences Ethics Committee. Written informed consent was obtained from each participant prior to enrolment.

The data was statistically processed by using Stata 12.1 (Stata Corp, College Station, Texas).

\section{RESULTS}

From a total of 150 patients, 86 patients (57.3\%) complained of discomfort of the lower respiratory tract, and a disorder of respiratory function was diagnosed by pulmonologists. Bronchial asthma was diagnosed and previously treated by a pulmonologist in $59(39.33 \%)$ of patients. Of the 86 patients with symptoms of lower respiratory tract, 38 patients $(44.18 \%)$ had a diagnosis of AERD.

The average age of the 150 patients was $52.83 \pm 12.28$ years. The youngest patient was 22 years old and the oldest had 83 years. There were seventy-five women (75) and the same number of men. Sex ratiowas 50\% women and 50\% men (1: 1).

Of the 150 patients with sino-nasal polyposis $38(25.33 \%)$ patients had AERD. Their average age was $47.24 \pm 13.35$, the youngest was 22 years old and the oldest 67 . Of the 38 patients 14 were male and 24 were female. Sex ratio was 1: 1.7 in favor of women. Positive test for inhalatory allergens in patients with AERD was registered in 18/38 (47.37\%). The test for eosinophils in the nasal secretion was positive in 28/38 patients (73.68\%). Non-allergic eosinophilic rhinitis (NARES) was present in 9/38 (23.68\%) patients. 
Nasal swab for bacteria and fungi was positive in $24 / 38$ patients (63.16\%) and in all the findings the dominant bacteria was Stapyloccocus aureus.

In the population with CRSwNP, SIA positive test was registered in 40/150 (30.66\%) patients, findings of eosinophils in the nasal discharge in 90/150 (60\%) patients, and nasalswab for bacteria and fungi was positive in 61/150 (40.66\%) cases. Non-allergic rhinitis with eosinophilia was registered in $62 / 150(41.33 \%)$ patients. There is a statistically significant difference between patients with AERD and the total number of patients with polyps with positive prick test $(p=0.013)$ and a positive test for nasal swabs $(p=0.012(p<0,05)$. However, there is no statistically significant difference in findings of positive eosinophils in the nasal secretions $\mathrm{p}=0.119(\mathrm{p}>0.05)$ (Fig. 1).

At the initial endoscopic examination of patients, second degree nasal polyposis was diagnosed in 14/38 (63.16\%) patients and the third level of polyps in 24/38 patients (36.84\%). After one year of therapy in 1/38 (2.63\%) patients polyps of I degree were registered, in 23/38 (60.53\%) patients - II degree, and in 14/38 (36.84\%) patients III degree nasal polyps was diagnosed.

In the total number of patients with nasal polyps, endoscopic findings before treatment were: I degree of nasal polyps in 78/150 (52\%) patients, II degree in 57/150 (38\%) patients and III degree of nasal polyps in $15 / 150(10 \%)$ of patients. After a year of therapy in $29 / 150$ (19.33\%) patients (pts) polyps was not found, while in 121/150 (80.66\%) patients polyps were diagnosed as (I degree polyps in 87/150 (58\%) pts, II in 29/150 (19.33\%) pts, III degree in 5/150 (3.33\%) pts (Figure 2). There is no statistically significant difference in the presence of II and III degree of polyposis in endoscopic findings before the initiation of therapy among patients with AERD and the total number of patients with nasal polyps $(\mathrm{p}=0.89$ and $\mathrm{p}=0.217, \mathrm{p}>0,05)$, but 
there is a statistically significant difference for I degree polyps $(p=0.042, \mathrm{p}<0,05)$ in those patients. After one year of therapy, endoscopic findings between these two groups are statistically significantly different $(\mathrm{p}=0.0217, p<0.05)$.

During one year, with the first therapy given at the beginning of the treatment, 10/38 $(26.32 \%)$ patients required another round of a twenty-day systemic corticosteroid therapy, a total of two rounds during the year. A total of three rounds during the year received 28/38 (73.68\%) patients. No patient was left on just one round of systemic corticosteroids.

In the total population of patients with CRSwNP, 36\% (54/150) pts received one round of therapy, $38.66 \%(58 / 150)$ pts two rounds and $25.33 \%$ (38/150) pts received three rounds.

There is a statistically significant difference between groups of patients with AERD polyps and the total number of patients with nasal polyps (CRSwNP) $(p=0.006, p<0.05)$.

Data on previous operations of nasal polyps was registered in 21/38 (55.26\%) of AERD patients. The largest number of repeated earlier operations, whether in the FESS surgery or simple polypectomy is four, and it was registered in one patient $1 / 38(2.63 \%)$, while three previous operations were registered in $7 / 38(18.42 \%)$ pts, two in $7 / 38(18.42 \%)$ pts and one in 6/38 (15.78\%) pts. One year after therapy, 29/38 (76.32\%) patients opted for FESS surgery. Of all the patients suffering from CRSwNP 34/150 (22.66\%) pts opted for surgical treatment. There is a statistically significant difference in the decision on the operational treatment between the two groups for $(\mathrm{p}=0, \mathrm{p}<0.05)$. 


\section{Cupic et al}

\section{DISCUSSION}

The concept of a single airway and the connection of airway diseases "remodeling" of the upper and lower respiratory tract, is confirmed by numerous studies, although it has been known for over two millennia. Lichtenstein picturesquely expressed it in the statement that "the nose is a part of the lung that is available to the finger" and Galen, in the second century stated that "frequent cleaning of the nasal discharge frees the lungs." The best example for this is the association of allergic diseases of the respiratory tract which manifests as chronic allergic respiratory syndrome - allergic rhinitis and asthma, and the occurrence of chronic rhinosinusitis with or without polyps and bronchial asthma. This also confirms the existence of one of the most severe forms of the disease, ie. remodeling of the airway: aspirin exacerbated respiratory disease (AERD) or ASA Syndrome $(1,2,3)$.

During the research, $86 / 150(57.3 \%)$ pts complained to the problems with lower respiratory tract. In these pts the respiratory function disorder was diagnosed by pulmonologist by functional diagnostic tests - body plethysmography and spirometry. Among these patients, 59 (39.33\%) patients had already been diagnosed and treated for bronchial asthma. Prieto et al. (9), find bronchial hypereactivity (BHR) in 35\% of patients with nasal polyps without clinically manifested asthma. Klossek et al (10), discover episodes of disrupted respiratory lung function in patients with nasal polyps in $42 \%$, and bronchial asthma in $26 \%$ of cases. Settipan $(11,12)$ states in his epidemiological study that $10 \%$ of asthmatic patients, who have an associated nasal polyps, at the same time develop polyps and asthma, and the others develop nasal polyps first, which are then followed by asthma (in a period of 2 to 12 years). Larsen (13) confirmed his claims. Our findings in the population tested for nasal polyps confirmed the association of nasal 
polyps and asthma (57.3\% bronchial hypereactivity and 39.33\% diagnosed asthma), which is consistent with previously published results.

CRSwNP occurs in 5\% of patients with atopic form of bronchial asthma and in $13 \%$ of patients with non-atopic asthma $(9,10,13)$. In the group of patients with severe asthma, among the most difficult to control is considered to be aspirin-induced asthma (AIA), in which the nasal polyps occurs in $36 \%-60 \%$ of patients (10).

In our study of 150 patients 38/150 (25.33\%) patients were diagnosed with AERD. Out of 38 patients 14 were men and 24 were women. Sex ratio was 1: 1.7 in favor of women. The mean age of patients was 47 . Our findings confirm studies alleging that in a population of patients with nasal polyps $8-26 \%$ of patients have aspirin intolerance. It also shows that in the total population of patients with CRSwNP, AERD polyps occurs earlier and is dominant in females. A typical patient with aspirin intolerance, asthma and nasal polyps (aspirin exacerbated respiratory disease (AERD) or ASA Syndrome) is a women of 30-40 years of age - up to two times more often than men as their prevalence is growing over 40 years of age $(10,13)$. Therefore, many authors believe that nasal polyps in middle aged women may indicate aspirin sensitivity and state that they are 1.6 times more likely to develop asthma and 2.7 times more likely to develop allergic rhinitis (13).

A positive skin prick test for standard inhaltory allergens was detected in $47,37 \%$, patients, which is consistent with studies of BergesGimeno et al., who register positive skin prick test in $34 \%$ to $64 \%$ of pts $(3,11,12,14)$. Although there have been earlier claims that the NARES precedes AERD, these studies question that claim, and we registered it in only $23.68 \%$. The main characteristic of nasal polyps in patients with asthma or AIA is that these are more difficult clinical forms of diffuse nasal polyps, with vast eosinophilia and hypertrophy of the 


\section{Cupic et al}

mucosa. Eosinophil count was 15 times higher in AIA patients than in patients with nasal polyposis without asthma and 4 times that in ATA. Eosinophilia in nasal discharge was reported in $73.68 \%$ of patients, as well as in studies of Bochenek et al, who registered it in $90 \%$ of pts, and also found it in high percentage in blood and sputum (15). In our study there were no statistically significant differences in the positive findings of eosinophils in the nasal secretion in population with nasal polyps and AERD, which can also be explained by the increased number of eosinophilic positive polyps in our population, according to the claims of Lacroix JS et al. (16) (Figure 1).

Nasal polyps and asthma in aspirin exacerbated airway diseases or earlier called ASA syndrome, have a long and difficultcourse, despite avoiding NSAIDs. Most patients suffer from severe obstruction of the nose, postnasal secretion and loss of smell. They are often complicated by infectious bacterial rhinosinusitis with the need for intensive antibiotic therapy, 5-6 times a year. Recent studies of Bachert et al $(17,18)$ have shown that Staphylococcus aureus enterotoxin participates in the pathogenesis of a group of patients with nasal polyps by superantigen mechanisms.

The presence of specific IgE detected in multiclonalIgE response in the nasal polyps (IgE antibodies to Staphylocoocus aureus enterotoxin (SAEs)) was found in 30-50\% of patients with nasal polyps and about $60-80 \%$ of patients with associated asthma $(18,19,20)$. A recent prospective study confirms the colonization of middle nasal meatus by Staphylocoocus aureus in $63.6 \%$ of patients with nasal polyps, and their connection with the $\operatorname{IgE}$ antibodies to the enterotoxin. Bernsein (5) finds in the $50 \%$ to $60 \%$ of cases the colonization of the middle nasal meatus with Staphylocoocus aureus, in the population of patients with nasal polyps. In our study, $63.16 \%$ of patients had a positive bacterial swab. In all patients, Staphylocoocus aureus was 
isolated, while other bacteria were not found (Figure 1). There is a significantly positive number of bacterial swabs on Staphylocoocus aureus in AERD polyps, in relation to the total number of patients suffering from CRSwNP $(p=0.012, p<0.05)$, which confirms earlier claims.

Endoscopic findings of third degree nasal polyps in $63.16 \%$ pts at the beginning of treatment and of second degree in $36.84 \%$ pts, confirm that AERD is phenotypically the most severe form for treatment, with fine-grained, cluster formation, very tough and hard for extraction, covered with white-yellowish secretion. When comparing the value of endoscopic findings before therapy among patients with AERD and the total number of patients with nasal polyps, there is no statistically significant difference in the presence of II and III degree of polyps ( $\mathrm{p}=0.89$ and $\mathrm{p}=0.217, \mathrm{p}>0.05$ ). There is, however, a statically significant difference for I degree polyps $(\mathrm{p}=0.042, \mathrm{p}<0.05)$. First degree polyps was not detected among AERD patients, indicating that AERD is most frequently found in II and III degree polyps (Figure 2).

AERD requires intensive, short-term oral corticosteroid therapy, followed by long-term topical corticosteroid therapy. It is necessary to continuously control the patients, at least once a month at the beginning of treatment and every three months later with the use of antibiotics, leukotrienes, anti-IL-5 therapy, and aspirin desensitization in doses $100-1300 \mathrm{mg}$ of aspirin. The mode of administration is intranasal or oral $(21,22,23)$. Sadly, aspirin desensitization is not implemented in our country as well as the confirmation of the diagnosis of aspirin intolerance with provocative tests. The study of Scadding GK et al. examined the impact of topical lysine aspirin in one nostril and the placebo in the other, in thirteen patients with bilateral polyps resistant to corticosteroid therapy. The result of the study was delayed recurrence of polyps and the absence of symptoms in 8 patients for a period of 15 months follow-up. Endoscopically, first degree polyps were diagnosed (24). 


\section{Cupic et al}

The confirmation of continuous treatment is the result of disease control compared to initial size of nasal polyps. In fact, at the end of the first year of study, in one patient first degree polyps $(2.63 \%)$ were noted, which was a significant reduction, $60.53 \%$ pts had second degree polyps, while $36.84 \%$ pts had third degree nasal polyps, which is considerably less than at the beginning of the study (Figure 6). There was a statistically significant reduction of polyps and better therapeutic response among the total population of patients with polyps, in contrary to pts with AERD polyps $(\mathrm{p}=0.0217, \mathrm{p}<0.05)$. This indicates there is resistance to therapy in certain number of pts with AERD and a weaker the therapeutic response (Fig. 2).

The reccurence of polyps, as the most characteristic feature of the disease, is great, so the need for systemic corticosteroid therapy as "conservative polypectomy" is common. The study of the European Network of aspirin-induced asthma (AIAN) is characterized by 500 aspirinsensitive patients, showed that $51 \%$ of patients require long-term systemic corticosteroid therapy, with inhalatory corticosteroids in average doses of $8 \mathrm{mg}$ per day of prednisone, to control the symptoms of the disease $(1,21,25,26)$.

In a similar study of 300 AIA patients in the USA, systemic corticosteroids have been used for a short period in $45 \%$ of patients and these patients had an average of 5.5 episodes of rhinosinusitis per year (27). In our research during one year of follow-up, none of the patients remained treated with one round of systemic corticosteroid therapy, which lasted 20 days, with daily continuous use of nasal corticosteroids. 10/38 (26.32\%) patients required another round of corticosteroid therapy, a total two rounds during the year. Three rounds during the year received 28/38 (73.68\%) of patients. This explains the high statistically significant difference in terms of a large number of repeated therapies in patients with AERD, compared to patients with nasal polyps. 
The high degree of recurrence of the disease and the need for surgical intervention is explained by the fact that during the first contact with a patient $21 / 38(55.26 \%)$ of patients had previously undergone surgery. Larsen, Tos et al. state that the recurrence rate of polyps is $75 \%$, while Rombaux et al. in $40,9 \%$ pts $(21,28,29)$. The largest number of repeated earlier operations, whether in the FESS surgery or simple polypectomy is 4 , registered in one patient $1 / 38(2.63 \%)$, while three operations previously had $7 / 38(18.42 \%)$ pts, two $7 / 38(18.42 \%)$ pts and one $6 / 38$ $(15.78 \%)$ pts.

It is believed that every patient with AERD has an average of more than three FESS surgeries during their lifetime, while other references state that every five years from the diagnosis of disease an average of one operation is performed (27). Because of constant complaints followed by worsening of asthma, regardless of the potentialreduction of nasal polyps which are recorded in AERD one year after conservative treatment, $76.32 \%$ of patients opted for operational procedure at the end of one year of conservative treatment. Another confirmation that the AERD is the most severe form of polyposis for treatment, with a high incidence of patients opting for surgical treatment is a statistically significant number of patients who choose surgery, in relation to total number of cases that is $22.66 \%$.

\section{CONCLUSION}

CHRwNP in AERD, is the most severe form of nasal polyps, with statistically significant presence of difficult forms of polyps (second and third degree), as well as the need for larger number of repeated rounds of systemic corticosteroids during one year and the worst result of treatment with a large number of residual polyps, as well as a statistically large number of 
decision on further surgical treatment. The degree of disease recurrence rate and resistance to therapy of this phenotypic form is manifested in a statistically significant, greater number of repeated (corticosteroid) rounds during treatment, as well as in the number of previously performed operations and decisions about new surgeries. A statistically significant difference of this phenotypic form of nasal polyps can be also found in laboratory findings of positive nasal swabs for Staphylocoocus aureus and positive prick test with standard inhaltory allergens, while there is no statistically significant difference in the findings of eosinophils in the nasal secretions.

\section{AUTHORS' NOTE}

All the authors of the manuscript have participated in the concept and design, analysis, drafting and revising of the manuscript. Each author listed on the manuscript has seen and approved the submission of this version of the manuscript and takes full responsibility for the manuscript. The authors of this paper have no any potential conflict of interest. 


\section{REFERENCES}

1. Kowalski ML, Makowska JS, Blanca M. Hypersensitivity to nonsteroidal antiinflammatory drugs (NSAIDs) - classification, diagnosis and management: review of the EAACI/ENDA and GA2LEN/HANNA. Allergy 2011; 66: 818-29.

2. Szczeklik A, Niżankowska-Mogilnicka E, Sanak M. Hypersensitivity to Aspirin and Non-Steroidal AntiinflammatoryDrugs.In:Adkinson:Middleton'sAllergy:Principles and Practice, 7th ed.2009; Vol2 Sec.D Ch. 69: 1227-39.

3. Berges-Gimeno M., Simon R.A., Stevenson D.D.: The natural history and clinical characteristics of aspirin exacerbated respiratory disease. Ann Allergy Asthma Immunol 2002; 89: 474-8.

4. Szczeklik A., Sanak M.: The broken balance in aspirin hypersensitivity. Eur J Pharmacol 2006; 533: 145-55.

5. Bernstein JM. The Inflammatory Process in Nasal Polyposis: Genetics, MolecularBiology, and Electrophysiology. Önerci M, Ferguson BJ. Nasal poyposis. Springer-Verlag Berlin Heidelberg 2010; 1: 27-34.

6. Loehrl TA, Ferre RM, Toohill RJ, Smith TL. Longterm asthma outcomes after endoscopic sinus surgery in aspirin triad patients. Am J Otolaryngol 2006; 27: 154-60.

7. Poetker DM, Mendolia-Loffredo S, Smith TL. Outcomes of endoscopic sinus surgery for chronic rhinosinusitis associated with sinonasal polyposis. Am J Rhinol1 2003; 1: 84-8.

8. Robinson JL, Griest S, James KE, Smith TL. Impact of aspirin intolerance on outcomes of sinus surgery. Laryngoscope 2007; 117: 825-30. 
9. Prieto L, Seijas T, Gutiérrez V, Uixera S, Bruno L, López R. Exhaled nitric oxide levels and airway responsiveness to adenosine $5 \not$-monophosphate in subjects with nasal polyposis. Int Arch Allergy Immunol 2004; 134: 303-9.

10. Klossek JM, Neukirch F, Pribil C, Jankowski R, Serrano E, Chanal I. Prevalence of nasal polyposis in France: A crosssectional, case-control study. Allergy 2005; 2: 233-7.

11. Settipane G. Epidemiology of nasal polyps. In: Settipane G, Lund VJ, Bernstein JM, Tos M., editor. Nasal polyps: epidemiology, pathogenesis and treatment. Rhode Island: Oceanside Publications 1997; 2: 17-24.

12. Settipane GA, Chafee FH, Klein DE. Aspirin intolerance. II. A prospective study in an atopic and normal population. J Allergy ClinImmunol 1974; 4: 200-4.

13. Larsen K. The clinical relationship of nasal polyps to asthma. In: Settipane G, Lund VJ, Bernstein JM, Tos M, editor. Nasal polyps: epidemiology, pathogenesis and treatment. Rhode Island: Oceanside Publications.1997; 1: 97-104.

14. Chafee F, Settipane GA. Aspirin intolerance. I. Frequency in an allergic population. J Allergy ClinImmunol 1974; 53: 193-9.

15. Bochenek G., Nizankowska E., Szczeklik A.: The atopy trait in hypersensitivity to nonsteroidal anti-inflammatory drugs. Allergy 1996; 51: 16-23.

16. Lacroix JS, Zheng CG, Goytom SH. Histological comparison of nasal polyposis in black African, Chinese and Caucasian patients. Rhinology 2002; 40: 118-21.

17. Bachert C, Gevaert P, Holtappels G, Johansson SG, van Cauwenberge Pl. Total and specific IgE in nasal polyps is related to local eosinophilic inflammation. J Allergy ClinImmunol 2001; 4: 607-14. 
18. Bachert C, Gevaert P, Howarth P, Holtappels G, van Cauwenberge P, Johansson SG. IgE to Staphylococcus aureus enterotoxins in serum is related to severity of asthma. J Allergy ClinImmunol 2003; 5: 1131-2.

19. Perez-Novo CA, Kowalski ML, Kuna P, Ptasinska A, Holtappels G, van Cauwenberge P. Aspirin sensitivity and IgE antibodies to Staphylococcus aureus enterotoxins in nasal polyposis: studies on the relationship. Int Arch Allergy Immunol 2004; 3: 255-60.

20. Conley DB, Tripathi A, Ditto AM, Reid K, Grammer LC, Kern RC. Chronic sinusitis with nasal polyps: Staphylococcal exotoxin immunoglobulin E and cellular inflammation. Am J. Rhinology 2004; 18: 273-8.

21. Lund VJ. Where Are We in the Medical Treatment of Nasal Polyps. Önerci M, Ferguson BJ. Nasal poyposis. Springer-Verlag Berlin Heidelberg 2010; 1: 239-48.

22. Gosepath J, Schafer D, Mann WJ. Aspirin sensitivity: long term follow-up after up to 3 years of adaptive desensitization using maintenance dose of $100 \mathrm{mg}$ of aspirin a day. Laryngorhinootologie 2002; 81:732-8.

23. Stevenson DD, Simon RA. Selection of patients for aspirin desensitization treatment. J Allergy Clin Immunol 2006; 118: 801-4.

24. Abhi A. Parikh, Glenis K. Scadding. Intranasal lysine-aspirin in aspirin-sensitive nasal polyposis: a controlled trial. The Laryngoscope. August 2005; Volume 115; 8: 1385-90.

25. Bellussi LM. Relapses After Surgery and Their Prevention. Nasal polyposis. Önerci M, Ferguson BJ. Nasal poyposis. Springer-Verlag Berlin Heidelberg 2010; 2: 119-98.

26. Nizankowska E., Duplaga M., Bochenek G.: on behalf of the AIANE project. Clinical course of aspirin-induced asthma, results of AIANE. In: Szczeklik A., Gryglewski R., 


\section{Cupic et al}

Vane J., et al ed. Eicosanoids, aspirin and asthma, New York: Marcel Dekker; 1998; 1: 451-72.

27. Dalziel K, Stein K, Round A, Garside R, Royle P. Systematic review of endoscopic sinus surgery for nasal polyps. Health Technology Assessment 2005; 7: 17-69.

28. Larsen K, Tos M. A long-term follow-up study of nasal polyp patients after simple polypectomies. Eur Arch Otorhinolaryngol 1997; 254: S85-8.

29. Rombaux P, De Toeuf C, Hamoir M, Eloy P, Bertrand B. Sinus-nasal polyposis: one-year outcome after endoscopic sinus surgery followed by topical corticosteroid therapy in 72 patients. Ann Otolaryngol Chir Cervicofac 2001; 118: 291-8. 


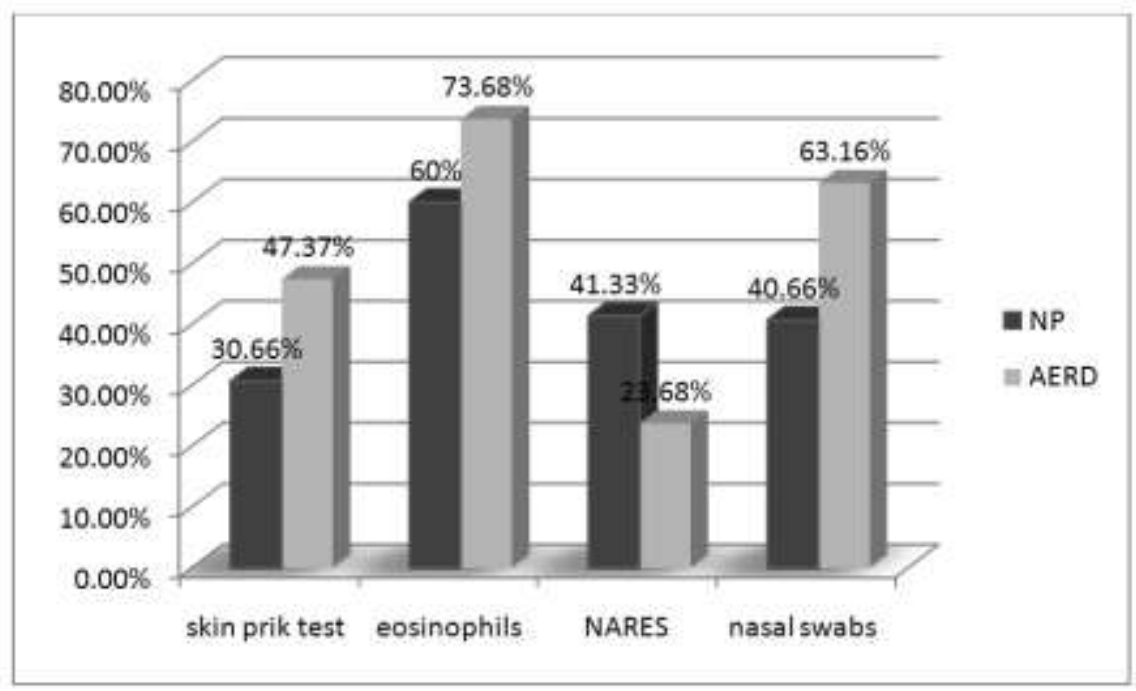

Fig. 1: Positive skin prick test findings: inhalalatedalergens, eosinophils in the nasal discharge, nasal swabs and the existence of non-allergic eosinophilic rhinitis among patients with nasal polyps and polyposis with AERD.

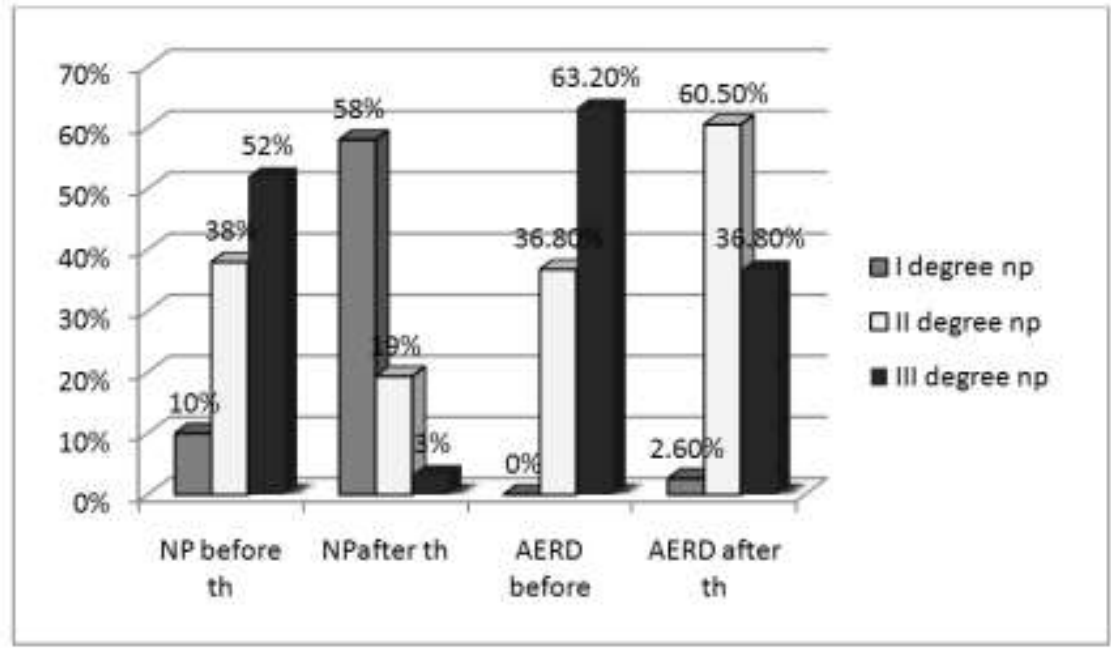

Fig. 2: Endoscopic findings in the total number of patients suffering from sino-nasal polyposis and polyposis with AERD before and after treatment for a period of one year. 